\title{
Sickness certificates: what information do they provide about rehabilitation?
}

\author{
Emma Nilsing Strid, Elsy Söderberg and Birgitta Öberg
}

\section{Linköping University Post Print}

\section{Tweet}

N.B.: When citing this work, cite the original article.

Original Publication:

Emma Nilsing Strid, Elsy Söderberg and Birgitta Öberg, Sickness certificates: what information do they provide about rehabilitation? 2014, Disability and rehabilitation, (36), 15, 1299-1304.

http://dx.doi.org/10.3109/09638288.2013.836252

Copyright: Informa Healthcare

http://informahealthcare.com/

Postprint available at: Linköping University Electronic Press

http://urn.kb.se/resolve?urn=urn:nbn:se:liu:diva-89837 


\begin{abstract}
Purpose: To investigate whether patients are prescribed rehabilitation early in a new sick leave period, and whether this prescription is associated with sex, age, diagnosis, description of functioning, and affiliation of certifying physician.

Method: A cross-sectional study using data from sickness certificates issued during a total sick leave period, collected consecutively during 2 weeks in 2007 in Östergötland County, Sweden. Rehabilitation prescribed in the first certificate or within 28 days after the start of sick leave was defined as early rehabilitation.

Results: Musculoskeletal diseases (MSD) were the largest diagnostic group, followed by mental disorders (MD). The mean duration of sick leave was 94 days (SD 139), longest for MD patients. Early rehabilitation was prescribed in $27 \%$ of all certificates and in $45 \%$ of certificates for MSD and MD diagnoses. Logistic regression analysis indicated that prescription of early rehabilitation was associated with certificates issued for MSD and MD, youngest patients, and certificates issued by primary health care physicians. The final model explained $29 \%$ of variation in the prescription of early rehabilitation.

Conclusions: There is a modest prescription of early rehabilitation in sickness certificates, based on younger age and MSD or MD diagnosis. This indicates that patients' rehabilitation needs may not have been identified.
\end{abstract}




\section{Background}

Sick leave rates vary over time, but long-term sick leave is still high in many Western European countries [1]. Sickness benefits are sanctioned for individuals who are unable to manage work demands owing to a disease or injury. In Sweden and many other countries, physicians are responsible for certifying sick leave after a period of self-certification ranging from no day in Denmark and Germany to seven days in Sweden and the UK [2]. The physician has to determine whether a disease or injury is present and whether this disease or injury impairs the patient's functioning to the extent that work ability is also reduced in relation to the demands at the patient's current work or in case of long-term sick leave, to other available jobs at the labour market $[2,3]$. Another main sickness certification task is to determine the need for medical investigations and interventions, such as rehabilitation [2]. Thus, both the sickness certification and the rehabilitation process depend initially on the physician and the physician's cooperation with other actors within the health care system, the social insurance office, the workplace and the patient $[2,4]$. In Sweden, the employer is responsible for providing workplace rehabilitation and work modification; and the social insurance office coordinates the needed rehabilitation interventions (SFS 2010:110, chapter 30). The sickness certificate provides an important informational basis for decisions regarding entitlement to sickness benefits, as well as return to work measures conveyed between the healthcare provider, the social insurance office, and the employer [2].

Early assessments of patients' functioning with structured screening for risk factors and questions about working conditions can help physicians to plan each patient's individually tailored interventions and return to work (RTW) [5, 6]. Rehabilitation interventions suggested to improve RTW are often multifaceted including cooperation between different healthcare professionals and contact with the workplace [7-10]. The timing is also important, and some 
studies have shown that early rehabilitation interventions are effective in supporting RTW [7, $10,11]$. The time frames constituting "early" vary though in the studies. When evaluating which rehabilitation interventions patients actually receive, differences based on patients' diagnoses, sex, and age are observed [11, 12]. Rehabilitation interventions are primarily awarded to younger patients $[12,13]$.

Physicians have reported problems with suggesting a plan of action during sick leave [14] and with ascertaining the ideal time to start rehabilitation [15]. A common attitude among interviewed physicians was that the best results are achieved with early rehabilitation; however, some found it time-consuming to evaluate the need for rehabilitation and opined that it might be futile to spend resources on rehabilitation when there is often a risk of unemployment [15]. Physicians have also reported that they seldom initiate contact with other healthcare professionals [16] or employers regarding patients' work problems [15-18], which may have implications for the patient regarding work modifications and RTW [18]. When direct contact with employers is sparse [15-18] and communication with social insurance officers is problematic [19, 20], information about patient's functioning and intervention needs may be primarily conveyed through sickness certificates. This information has implications for decisions regarding sickness benefits, as well as for the coordination of RTW interventions $[2,21]$. The objective of this study was to investigate whether patients are prescribed rehabilitation early in a new sick leave period, and whether the prescription is associated with patient's sex, age, diagnosis, description of functioning, and affiliation of the certifying physician.

\section{Methods}

Study design and sample 
The design is a cross-sectional study using data from sickness certificates issued by physicians for a new sick leave period. This study was approved by the Research Ethics Committee of the Faculty of Health Sciences of Linköping University, Sweden.

All of the new sickness certificates delivered to the social insurance offices in Östergötland County, Sweden (population 420,809) were collected consecutively during 2 weeks in September 2007 ( $n=497)$ and followed until the end of the current sick leave period. Therefore, any incoming certificate that prolonged the sick leave was also collected until the current sick leave period ended or up to one year. After excluding 22 certificates because they did not certify a new sick leave period $(n=16)$, or because of death $(n=4)$ or incorrect personal identity number $(n=2), 475$ new sickness certificates were included in the analysis. When including certificates prolonging the sick leave, the total sample size was 1,311 sickness certificates. The sample does not include the first 7 days of self-certification or the first two weeks of sick pay from the employer. By the time of data collection, there were no timelines for review of eligibility or maximum length of sick leave in Sweden.

Information collected from the first sickness certificates issued for a new sick leave period included the following factors: patient's sex; patient's age $(\leq 24,25-34,35-44,45-54$, or $\geq 55$ years); main diagnosis underlying the cause of sick leave, coded according to the International Classification of Diseases and Related Health Problems, Tenth Revision (ICD-10); affiliation of the certifying physician [primary health care (PHC), occupational health service (OHS), hospital, or private clinic]; and certified part-time sick leave (yes/no). The ICD-10 codes were categorised as follows: $\mathrm{F}$ into mental disorders (MD), $\mathrm{M}$ into musculoskeletal diseases (MSD), I and J into cardiorespiratory diseases (CR), and the remaining codes (A-E, G, H, K$\mathrm{N}, \mathrm{O}-\mathrm{Z}$ ) into the diagnostic group Other Diagnoses. Although this categorisation has been 
used previously [22], respiratory and cardiovascular diseases were treated as a single category in the present study.

Information about length of sick leave per patient was obtained by calculating the number of sick leave days prescribed in the first sickness certificate, as well as in prolonged certificates. Days of partial absence due to sickness were combined (e.g., 2 days of 50\% sick leave were counted as one full day).

The text written in the certificates in response to the question "How does the disease limit the patient's ability/activity", constituting the most important basis for decisions on entitlement to sickness benefits, was collected from the first sickness certificate in the sick leave period. This free text was analysed deductively using content analysis [23, 24], with the International Classification of Functioning, Disability and Health (ICF) as a theoretical framework and classification [25]. The text was read and meaningful concepts were identified and linked to the different components of ICF: body functions/structures and activities/participation [25, 26]; insufficient text was entered as "no description" [26]. The analysis was conducted by two independent researchers and disagreements were solved in consensus discussions in the research group. The analysis is described in detail in a previous paper [27]. Although certificates might provide information according to more than one component, a hierarchical variable was used in the logistic regression analysis.

The prescription "intervention essential for recovery of ability" in sickness certificates includes free text, which was collected from the initial certificate issued during a new sick leave period, as well as from incoming certificates that prolonged the current sick leave period. To structure this information, it was categorised as rehabilitation, medical 
intervention, or no intervention. Rehabilitation comprised physiotherapy, counselling (i.e., conversation therapy), occupational therapy, or referral to a rehabilitation clinic or occupational health service. A cut-off point of $\leq 28$ days of sick leave was defined as early prescribed rehabilitation. This cut-off was chosen based on the common way to divide patients with low back pain with regard to duration of pain: acute $(<4$ weeks), sub-acute (412 weeks), and chronic pain (> 12 weeks) [28], but also according to the Swedish social insurance office's categorisation of short-term sick leave cases (0-28 days) [29]. Rehabilitation prescribed in the first certificate or within 28 days after the beginning of the sick leave period was defined as early rehabilitation, and rehabilitation prescribed after 28 days was considered late rehabilitation. Prescriptions of medication or advice were defined as medical interventions, and no intervention refers to certificates without a prescription of any intervention. Certificates prescribing both medical and rehabilitation interventions were placed in the category of rehabilitation.

The question in the certificate "are RTW measures needed?" is to be answered by selecting one of the following alternatives: Yes, No, Cannot be assessed for the moment, or In need of OHS. This information was collected from the first certificate issued during a new sick leave period, as well as from certificates prolonging the current sick leave period. The answers "No" and "Cannot be assessed for the moment" were combined into "No", and "Yes" and "In need for OHS" were combined into "Yes".

\section{Statistics}

Tests were two-sided, with a significance level of $p<0.05$. Descriptive analyses were calculated using proportions or means with standard deviations (SD) or 95\% confidence interval. Ninety-five percent confidence intervals (95\% CIs) were calculated for all odds 
ratios (ORs). Chi-squared tests were used for group comparisons of categorical variables. Student's $t$-test and one-way ANOVA with post hoc Bonferroni test were used for group comparisons of numerical variables. Measures of sick leave length were checked with nonparametric tests in addition to one-way ANOVA. A logistic regression analysis with the dependent variable prescription of early rehabilitation was conducted to assess the association between the dichotomous outcome and several independent variables (i.e. sex, age, diagnosis, description of functioning, and affiliation of certifying physician). Method entry was used. Statistical analyses were calculated using SPSS (version 19.0; SPSS Inc., Chicago, IL).

\section{Results}

As presented in Table 1, patients with MSD comprised the largest diagnostic group $(n=137)$, followed by those with MD $(n=80)$. Within the diagnostic group Other Diagnoses $(n=211)$, injuries $(n=58)$ and pregnancy-related disorders $(n=27)$ were most common. The mean age of patients was 45 years (SD 12.5). The majority of the certificates were issued for women (n = 293), even when pregnancy-related disorders were excluded. Certificates were issued primarily by physicians at hospitals and in PHC, and to a lesser extent at private clinics and the OHS. A description of functioning according to ICF was mainly provided regarding body functions, and less so regarding activity/participation. More than one-third of the first sickness certificates lacked a description of functioning (Table 1). RTW measures were proposed in $6 \%$ of the first certificates, and in $7 \%$ of certificates issued later in the sick leave period $(13 \%$ total).

Insert table 1 about here 
The mean duration of total certified sick leave period was 94 days (SD 139, median 36). Sick leave was longer in certificates issued for patients with MD (mean 126 days, SD 165) than in those issued for patients in the diagnostic group Other Diagnoses (mean 73 days, SD 116). Thirteen percent of the first sickness certificates were issued for part-time sick leave. Women were certified for part-time sick leave more often than men (16\% vs. $8 \%$, respectively, $p=$ 0.011). Part-time sick leave was certified more frequently by PHC physicians (19\%) and OHS physicians $(25 \%)$ than by physicians at hospitals $(6 \%)$ or private clinics $(8 \%)$.

\section{Prescribed interventions}

In $27 \%(\mathrm{n}=130)$ of all certificates, rehabilitation interventions (i.e., physiotherapy, counselling, occupational therapy, referral to OHS or rehabilitation clinic) were prescribed in the first certificate or within 28 days of sick leave in a prolonging certificate. These interventions are defined as early rehabilitation. Rehabilitation was prescribed later in the current sick leave period in an additional 37 certificates (within 29-418 days), defined as late rehabilitation. In the first certificate or within 28 days of sick leave, medical interventions were prescribed as the only mode of intervention in $39 \%(n=187)$, but in combination with rehabilitation in 264 certificates. There was no information about interventions in $26 \%$ of the certificates $(n=121)$ (Table 1$)$.

The prescribed interventions differed regarding type of diagnosis. Different diagnoses were represented in different age intervals (Table 2). Back pain was the most frequent diagnosis (41\%) in the diagnostic group MSD. Arthritis was more common in elderly patients, and soft tissue disorders were more common among younger patients. Within the diagnostic group MD, depressive disorders (46\%) were common in all age intervals, and were slightly more common among older patients. Younger patients were more often certified for sick leave 
because of reaction to stress and anxiety disorders (Table 2). As presented in table 3, early rehabilitation (mainly physiotherapy) was primarily prescribed in sickness certificates issued for back pain, soft tissue disorders, or other M-diagnoses. Certificates issued for arthritis and other joint disorders provided less information about prescribed interventions. Early rehabilitation (mainly counselling), was primarily prescribed in certificates issued for stress disorders (Table 3).

Insert tables 2 and 3 about here

Early rehabilitation was mainly prescribed in certificates issued for women $(p=0.022)$, the youngest patients, patients in the diagnostic groups MSD and MD, in certificates with a description of functioning according to the ICF activity/participation components, or in certificates issued by a PHC or OHS physician. A logistic regression model demonstrated that certificates issued for patients with MSD or MD, for the youngest patients, and by PHC physicians were associated with the prescription of early rehabilitation. The final model explained $29 \%$ of the variation in early rehabilitation prescription (Table 4).

Insert table 4 about here

\section{Discussion}

The main finding of this study was that early rehabilitation is prescribed in approximately one-fourth of the sickness certificates issued during a total sick leave period. Prescription of early rehabilitation was associated with certificates issued for MSD and MD, certificates issued to the youngest patients, and certificates issued by PHC physicians. 
The finding that early rehabilitation interventions were only prescribed in approximately onefourth of sickness certificates is in agreement with a concurrent study conducted in the same county council, which included patients with MSD and MD who were certified sick leave by PHC or OHS [11]. Wåhlin et al. observed that within three months, 45\% of these patients reportedly have received a combination of medical and rehabilitative intervention modalities, and $31 \%$ had also received work-related interventions [11]. When only MD and MSD were included in the current analysis, early rehabilitation was prescribed in $45 \%$ of the certificates issued for these two diagnostic groups. This finding indicates that the validity might be acceptable, and the modest prescription of early rehabilitation may reflect behaviour in practice. Accordingly, there is a risk that patients may not receive interventions according to their needs.

The severity of the disease, disability, and impaired work ability differ among the patients that different physicians encounter, and may affect the suggestion of intervention. Whether or not a disease hinders a patient from work depends mainly on the patient's activity limitation in relation to work tasks and work demands [30], as well as whether the employer is involved in enhancing RTW through changes in work tasks or workplace conditions [18]. In this study, being among the youngest patients was correlated with the likelihood of receiving a prescription for early rehabilitation. Similar findings are observed in studies that include longterm sickness absentees, which show an association of older age with either lack of a rehabilitation recommendation [13] or advocated vocational rehabilitation [12]. Although we identified that more women were prescribed early rehabilitation compared with men, this difference was not statistically significant in the logistic regression analysis. Neither age nor sex should influence the prescription of interventions; however, more emphasis may be put on improving younger patients' functioning and enhancing their return to work. One explanation 
may be the type of disease and its degree of severity, and we observed that different diagnoses were more or less frequent for patients of different ages (e.g., arthritis was more frequent in older patients, and soft tissue disorders were more frequent among younger patients). More patients with MSD or MD were prescribed early rehabilitation than medical intervention, while the converse was true for patients in the group Other Diagnoses. Rehabilitation may be looked upon as more important for patients with symptom-based diagnoses for which no clear medical treatment is offered. We found that approximately $60 \%$ of the sickness certificates issued for patients with low back pain or stress-related disorders were prescribed early rehabilitation. However, more than one-half of the certificates issued for arthritis diagnoses lacked information about interventions, and early rehabilitation was prescribed in less than one-fourth of these cases. Because exercise is the core treatment for osteoarthritis [31, 32], more prescriptions of physiotherapy might have been expected. We found that PHC physicians prescribed early rehabilitation more often than medical intervention, while hospital physicians had the opposite prescribing pattern. This finding may be partly explained by the different needs of patients that encounter PHC versus hospital physicians.

Because the goals of early rehabilitation are to address bio-psychosocial obstacles to recovery and RTW and to increase functioning [33, 34], the patient's functioning should provide the basis for rehabilitation decisions. In this study, the description of functioning was not associated with a prescription of early rehabilitation in comparison with diagnosis, age, and physician type. On the other hand, the description of functioning in sickness certificates needs improvement, including activity limitations or participation restrictions [35].

It is difficult to estimate a reasonable prescription of rehabilitation. Rehabilitation interventions may not be needed for everyone on sick leave; however, many patients may 
have had symptoms several years before the start of the sick leave [36]. Guidelines for the management of sick leave suggest that the most effective interventions are early, multidisciplinary, and include the workplace [10]. The timing of interventions is further emphasised by the associations observed between the development of chronic disability and 2- to 4-week delays in administration or access to medical treatment [37]. Chronic disability was associated with physicians with little experience in compensation systems, regardless of the severity of the disease or injury [37]. The modest prescription of early rehabilitation in sickness certificates may depend on several factors, such as lack of time or knowledge [15, 16]. A focus on early assessments of patients' functioning, including screening for risk factors and working conditions, is needed in order to facilitate tailored interventions and RTW $[5,6]$.

\section{Methodological considerations}

Strengths of this study are that it includes all of the sickness certificates that the social insurance office in Östergötland County, Sweden, received during the study period, and that these certificates were followed until the end of the current sick leave period or up to one year. The sample size and duration of sick leave were expected with regard to the trend of decreasing new sick leave spells. All of the variables were collected from sickness certificates; analyses are restricted to the information provided in these certificates and how this information was interpreted and categorised. We used a cut-off point of 28 days to define early rehabilitation; however, because rehabilitation was rarely prescribed later in the sick leave period, the cut-off point may not have been particularly important in this study. The logistic regression model explained $29 \%$ of the variation in early rehabilitation prescription, which indicates that other factors likely contribute to the explanation of the variation in prescription. Some of these other factors may be related to the physician, such as knowledge and resources, and some may be related to the patient. Categorisation into subgroups led to 
different sample sizes. The diagnostic group Other is a heterogeneous group consisting of a wide range of diagnoses, and the groups CR, OHS, and Private Clinics are based on small sample sizes. These certificates were included in the analyses because the study objective was to investigate the prescription of early rehabilitation in all certificates; however, the results based on these subgroups should be interpreted with caution. The duration of sick leave was based on the number of prescribed days of sick leave as a measure of the gathered individual illness burden [38]. We combined days of partial sick leave, as has been done previously [39]. However, there is a difference between being on partial or full-time sick leave, which must be considered particularly when analyzing the effects of interventions [38]. Finally and most importantly, because we have investigated information in sickness certificates regarding whether patients are prescribed rehabilitation early in their sick leave, we do not know what actually happens in practice and when, or what the choice of intervention is based on. However, sickness certificates are an important basis for decision-making regarding entitlement to sickness benefits, as well as a communicative tool for the coordination of RTW measures, and the results of this study should be interpreted from this perspective. If social insurance officers, employers, and patients do not receive information about which interventions are needed, they will have difficulties acting as expected. Additional studies are needed regarding how to develop stakeholder communication, and whether improved communication facilitates the performance of interventions and enhances RTW.

\section{Conclusion}

In conclusion, different rehabilitation needs are associated with various diagnoses, but the findings from this study show that early rehabilitation is modestly prescribed in sickness certificates. More than one-half of the certificates issued for MSD and MD did not include any prescription for early rehabilitation. The prescription of early rehabilitation was merely 
based on younger age and MSD or MD diagnoses, rather than on needs defined as functioning according to the ICF. This finding indicates that patients' rehabilitation needs might not have been identified when the sickness certificates were completed.

\section{Declaration of interest}

The authors have no conflicts of interest to declare. The study was supported by grants from the National Social Administration and the County Council in Östergötland, Sweden (grant number IHS 7/0728). All authors are responsible for the content and writing of the paper. 


\section{References}

1. OECD. Sickness, Disability and Work. Breaking the Barriers. A Synthesis of Findings across OECD Countries. OECD Publishing; 2010.

2. Wahlstrom R, Alexanderson K. Swedish Council on Technology Assessment in Health Care (SBU). Chapter 11. Physicians' sick-listing practices. Scand J Public Health Suppl 2004; 63:222-255.

3. Wynne-Jones G, Mallen CD, Main CJ, Dunn KM. What do GPs feel about sickness certification? A systematic search and narrative review. Scand J Prim Health Care 2010; 28:67-75.

4. de Boer WEL, Besseling JJM, Willems JHB. Organisation of disability evaluation in 15 countries. Prat Organ Soins 2007; 38:205-217.

5. Steenstra IA, Ibrahim SA, Franche RL, Hogg-Johnson S, Shaw WS, Pransky GS. Validation of a risk factor-based intervention strategy model using data from the readiness for return to work cohort study. J Occup Rehabil 2010; 20:394-405.

6. Shaw WS, van der Windt DA, Main CJ, Loisel P, Linton SJ. Early patient screening and intervention to address individual-level occupational factors ("blue flags") in back disability. J Occup Rehabil 2009; 19:64-80.

7. Hoefsmit N, Houkes I, Nijhuis FJ. Intervention Characteristics that Facilitate Return to Work After Sickness Absence: A Systematic Literature Review. J Occup Rehabil 2012; 22:462-477.

8. Kuoppala J, Lamminpaa A. Rehabilitation and work ability: a systematic literature review. J Rehabil Med 2008; 40:796-804.

9. van Oostrom SH, Driessen MT, de Vet HC, Franche RL, Schonstein E, Loisel P, van Mechelen W, Anema JR: Workplace interventions for preventing work disability. Cochrane Database Syst Rev 2009; CD006955.

10. Gabbay M, Taylor L, Sheppard L, Hillage J, Bambra C, Ford F, Preece R, Taske N, Kelly MP. NICE guidance on long-term sickness and incapacity. Br J Gen Pract 2011; 61:118-124.

11. Wahlin C, Ekberg K, Persson J, Bernfort L, Oberg B. Evaluation of Self-Reported Work Ability and Usefulness of Interventions Among Sick-Listed Patients. J Occup Rehabil 2013; 23:32-43.

12. Heijbel B, Josephson M, Vingard E. Implementation of a rehabilitation model for employees on long-term sick leave in the public sector: Difficulties, counter-measures, and outcomes. Work 2012; doi 10.3233/WOR-2012-1478.

13. Salmi P, Svedberg P, Hagberg J, Lundh G, Linder J, Alexanderson K. Outcome of multidisciplinary investigations of long-term sickness absentees. Disabil Rehabil 2009; 31:131-137.

14. Lofgren A, Hagberg J, Arrelov B, Ponzer S, Alexanderson K. Frequency and nature of problems associated with sickness certification tasks: A cross-sectional questionnaire study of 5455 physicians. Scand J Prim Health Care 2007; 25:178-185.

15. Edlund C, Dahlgren L. The physician's role in the vocational rehabilitation process. Disabil Rehabil 2002; 24:727-733.

16. Coole C, Watson PJ, Drummond A: Work problems due to low back pain: what do GPs do? A questionnaire survey. Fam Pract 2010; 27:31-37.

17. Timpka T, Hensing G, Alexanderson K. Dilemmas in sickness certification among Swedish physicians. Eur J Public Health 1995; 5:215-219.

18. Pransky G, Katz JN, Benjamin K, Himmelstein J. Improving the physician role in evaluating work ability and managing disability: a survey of primary care practitioners. Disabil Rehabil 2002; 24:867-874. 
19. Hussey S, Hoddinott P, Wilson P, Dowell J, Barbour R. Sickness certification system in the United Kingdom: qualitative study of views of general practitioners in Scotland. BMJ 2004; 328:88.

20. von Knorring M, Sundberg L, Lofgren A, Alexanderson K. Problems in sickness certification of patients: A qualitative study on views of 26 physicians in Sweden. Scand J Prim Health Care 2008; 26:22-28.

21. Soderberg E, Alexanderson K. Sickness certificates as a basis for decisions regarding entitlement to sickness insurance benefits. Scand J Public Health 2005; 33:314-320.

22. Martimo KP, Varonen H, Husman K, Viikari-Juntura E. Factors associated with selfassessed work ability. Occup Med (Lond) 2007; 57:380-382.

23. Downe-Wamboldt B. Content analysis: method, applications and issues. Health Care Women Int 1992; 13:313-321.

24. Hsieh HF, Shannon SE. Three approaches to qualitative content analysis. Qual Health Res 2005; 15:1277-1288.

25. World Health Organization. ICF - International classification of functioning, disability and health. Genève: World Health Organization; 2001.

26. Cieza A, Geyh S, Chatterji S, Kostanjsek N, Ustun B, Stucki G. ICF linking rules: an update based on lessons learned. J Rehabil Med 2005; 37:212-218.

27. Nilsing E, Soderberg E, Normelli H, Oberg B. Description of functioning in sickness certificates. Scand J Public Health 2011; 39:508-516.

28. Waddell G. The back pain revolution. 2nd ed. Edinburgh: Churchill Livingstone; 2004.

29. The Swedish Social Insurance Agency [Internet]. [Cited 2013 Aug 12]; Available from: http//www.forsakringskassan.se

30. Alexanderson K, Norlund A. Swedish Council on Technology Assessment in Health Care (SBU). Chapter 1. Aim, background, key concepts, regulations, and current statistics. Scand J Public Health Suppl 2004; 63:12-30.

31. The Swedish National Board of Health and Welfare [Internet]. National guidelines for musculoskeletal diseases. Stockholm: 2012. [Cited 2013 Aug 12]; Available from: http://www.socialstyrelsen.se/Lists/Artikelkatalog/Attachments/18665/2012-5-1.pdf

32. National Institute for Health and Clinical Excellence [Internet]. Osteoarthrities. The care and management of osteoarthritis in adults. London: 2008. [Cited 2013 Aug 12]; Available from http://www.nice.org.uk

33. Stucki G, Stier-Jarmer M, Grill E, Melvin J. Rationale and principles of early rehabilitation care after an acute injury or illness. Disabil Rehabil 2005; 27:353-359.

34. Waddell G, Burton AK. Concepts of rehabilitation for the management of common health problems. London: TSO; 2004.

35. Nilsing E, Soderberg E, Oberg B. Sickness certificates in Sweden: did the new guidelines improve their quality? BMC Public Health 2012; 12:907.

36. Heijbel B, Josephson M, Jensen I, Vingard E. Employer, insurance, and health system response to long-term sick leave in the public sector: policy implications. J Occup Rehabil 2005; 15:167-176.

37. Sinnott P. Administrative delays and chronic disability in patients with acute occupational low back injury. J Occup Environ Med 2009; 51:690-699.

38. Hensing G, Alexanderson K, Allebeck P, Bjurulf P. How to measure sickness absence? Literature review and suggestion of five basic measures. Scand J Soc Med 1998; 26:133-144.

39. Alexanderson K, Borg K, Hensing G. Sickness absence with low-back, shoulder, or neck diagnoses: an 11-year follow-up regarding gender differences in sickness absence and disability pension. Work 2005; 25:115-124. 
Table 1. Prescribed interventions, cross-tabulated by information provided in sickness certificates, row \% (n)

\begin{tabular}{lllll}
\hline Variable (n) & $\begin{array}{l}\text { No } \\
\text { intervention }\end{array}$ & $\begin{array}{l}\text { Medical } \\
\text { Intervention }\end{array}$ & $\begin{array}{l}\text { Early } \\
\text { Rehabilitation }\end{array}$ & $\begin{array}{l}\text { Late } \\
\text { Rehabilitation }\end{array}$ \\
\hline Sex (475) & & & & \\
Women (293) & $22(63)$ & $39(114)$ & $31(91)$ & $9(25)$ \\
Men (182) & $32(58)$ & $40(73)$ & $21(39)$ & $7(12)$ \\
Age interval (475) & & & & \\
$<24(26)$ & $15(4)$ & $35(9)$ & $50(13)$ & $0(0)$ \\
25-34 (90) & $23(21)$ & $37(33)$ & $34(31)$ & $6(5)$ \\
$35-44(108)$ & $31(33)$ & $41(44)$ & $19(21)$ & $9(10)$ \\
$45-54(112)$ & $23(26)$ & $37(41)$ & $31(35)$ & $9(10)$ \\
$>55(139)$ & $27(37)$ & $43(60)$ & $22(30)$ & $9(12)$ \\
Diagnosis (470) & & & & \\
MSD (137) & $26(36)$ & $19(26)$ & $45(61)$ & $10(14)$ \\
MD (80) & $11(9)$ & $34(27)$ & $45(36)$ & $10(8)$ \\
CR (42) & $21(9)$ & $67(28)$ & $7(3)$ & $5(2)$ \\
Other (211) & $31(65)$ & $49(103)$ & $14(30)$ & $6(13)$ \\
Functioning*(475) & & & & \\
Activity/participation & $17(23)$ & $34(45)$ & $37(49)$ & $13(17)$ \\
(134) & & & & \\
Body (276) & $18(50)$ & $42(115)$ & $32(88)$ & $8(23)$ \\
None (164) & $37(60)$ & $37(60)$ & $20(32)$ & $7(12)$ \\
Physician affiliation & & & & \\
(470) & & & & \\
PHC (201) & $16(32)$ & $36(73)$ & $41(82)$ & $7(14)$ \\
OHS (24) & $8(2)$ & $29(7)$ & $42(10)$ & $21(5)$ \\
Private (39) & $46(18)$ & $28(11)$ & $21(8)$ & $5(2)$ \\
Hospital (206) & $33(68)$ & $46(94)$ & $14(29)$ & $7(15)$ \\
\hline Total & $26(121)$ & $39(187)$ & $27(130)$ & $8(37)$ \\
\hline
\end{tabular}

Early rehabilitation is defined as the prescription of rehabilitation in the first certificate or within 28 days after the start of the current sick leave period. Late rehabilitation is defined as a prescription after 28 days of sick leave.

Abbreviations: MSD, musculoskeletal diseases; MD, mental disorders; CR, cardiorespiratory diseases; Other, other diagnostic groups; PHC, primary health care; OHS, occupational health service.

*The sum is more than $100 \%$ because some certificates provided a description of functioning categorised into more than one ICF component. 
Table 2. Type of diagnosis within the diagnostic groups musculoskeletal diseases (MSD) and mental disorders (MD), presented in different age intervals, column \% (n).

\begin{tabular}{|c|c|c|c|c|c|c|}
\hline Diagnosis & $\mathrm{N}$ & $\leq 24$ & $25-34$ & $35-44$ & $45-54$ & $\geq 55$ \\
\hline \multicolumn{7}{|l|}{ MSD } \\
\hline Arthrosis and other joint disorders & 34 & 0 & $17(4)$ & $5(1)$ & $18(7)$ & $44(22)$ \\
\hline $\begin{array}{l}\text { Dorsopathies and disorders of } \\
\text { synovium and tendon }\end{array}$ & 56 & 0 & $29(7)$ & $50(11)$ & 49 (19) & 38 (19) \\
\hline Other M-diagnoses & 47 & $100(2)$ & $54(13)$ & $45(10)$ & $33(13)$ & $18(9)$ \\
\hline Total & 137 & $100(2)$ & $100(24)$ & $100(22)$ & $100(39)$ & $100(50)$ \\
\hline \multicolumn{7}{|l|}{ MD } \\
\hline Depression & 37 & $33(2)$ & $38(8)$ & $52(13)$ & $46(6)$ & $53(8)$ \\
\hline Stress disorders & 33 & $50(3)$ & $57(12)$ & $28(7)$ & $39(5)$ & $40(6)$ \\
\hline Other F-diagnoses & 10 & $17(1)$ & $5(1)$ & $20(5)$ & $15(2)$ & $7(1)$ \\
\hline Total & 80 & $100(6)$ & $100(21)$ & $100(25)$ & $100(13)$ & $100(15)$ \\
\hline
\end{tabular}


Table 3. Prescribed interventions cross-tabulated by type of diagnosis within the diagnostic groups musculoskeletal diseases (MSD) and mental disorders (MD), row \% (n)

\begin{tabular}{|c|c|c|c|c|c|}
\hline Diagnosis & $\mathrm{N}$ & $\begin{array}{l}\text { No } \\
\text { intervention }\end{array}$ & $\begin{array}{l}\text { Medical } \\
\text { intervention }\end{array}$ & $\begin{array}{l}\text { Early } \\
\text { rehabilitation }\end{array}$ & $\begin{array}{l}\text { Late } \\
\text { rehabilitation }\end{array}$ \\
\hline \multicolumn{6}{|l|}{ MSD } \\
\hline $\begin{array}{l}\text { Arthrosis and other joint } \\
\text { disorders }\end{array}$ & 34 & 59 (19) & $15(5)$ & $18(6)$ & $12(4)$ \\
\hline $\begin{array}{l}\text { Dorsopathies and disorders of } \\
\text { synovium and tendon }\end{array}$ & 56 & $16(9)$ & $18(10)$ & $57(32)$ & $9(5)$ \\
\hline Other M-diagnoses & 47 & $17(8)$ & $23(11)$ & $49(23)$ & $11(5)$ \\
\hline Total & 137 & $26(36)$ & $19(26)$ & $45(61)$ & $10(14)$ \\
\hline \multicolumn{6}{|l|}{ MD } \\
\hline Depression & 37 & $8(3)$ & $41(15)$ & $35(13)$ & $16(6)$ \\
\hline Stress disorders & 33 & $12(4)$ & $27(9)$ & $58(19)$ & $3(1)$ \\
\hline Other F-diagnoses & 10 & $20(2)$ & $30(3)$ & $40(4)$ & $10(1)$ \\
\hline Total & 80 & $11(9)$ & $34(27)$ & $45(36)$ & $10(8)$ \\
\hline
\end{tabular}

Early rehabilitation is defined as a prescription of rehabilitation in the first certificate or within 28 days after the start of the current sick leave period. Late rehabilitation is defined as a prescription after 28 days of sick leave. 
Table 4. Association between the prescription of early rehabilitation and information provided in sickness certificates regarding sex, age, diagnosis, description of functioning, and affiliation of certifying physician.

\begin{tabular}{|c|c|c|c|c|c|}
\hline Independent variable & $\mathrm{B}(\mathrm{SE})$ & Wald & $\mathrm{df}$ & OR $(95 \% \mathrm{CI})$ & $\mathrm{P}$ \\
\hline \multicolumn{6}{|l|}{ Model Enter } \\
\hline Sex & \multicolumn{5}{|c|}{1} \\
\hline Women & $0.4(0.3)$ & 2.6 & & $1.5(0.9-2.5)$ & \\
\hline Age, years & & & 4 & & \multirow{6}{*}{$\begin{array}{l}0.001 \\
0.052\end{array}$} \\
\hline$\leq 24$ & $1.7(0.5)$ & 10.4 & & $5.5(2.0-15.3)$ & \\
\hline $25-34$ & $0.6(0.3)$ & 3.8 & & $2.0(1.0-3.9)$ & \\
\hline $35-44$ & $-0.1(0.4)$ & 0.1 & & $0.9(0.5-1.8)$ & \\
\hline $45-54$ & $0.6(0.3)$ & 3.2 & & $1.8(0.9-3.4)$ & \\
\hline$\geq 55$ & & & & 1 & \\
\hline Diagnosis & & & 3 & & \multirow{5}{*}{$\begin{array}{l}<0.001 \\
<0.001\end{array}$} \\
\hline MSD & $1.5(0.3)$ & 23.8 & & $4.5(2.5-8.3)$ & \\
\hline MD & $1.5(0.3)$ & 18.8 & & $4.5(2.3-8.8)$ & \\
\hline $\mathrm{CR}$ & $-1.0(0.7)$ & 2.5 & & $0.4(0.1-1.3)$ & \\
\hline Other & & & & 1 & \\
\hline Functioning & & & 2 & & \\
\hline Body & $0.2(0.3)$ & 0.5 & & $1.3(0.7-2.3)$ & \\
\hline Activity/participation & $-0.1(0.3)$ & 0.0 & & $0.9(0.5-1.7)$ & \\
\hline No description & & & & 1 & \\
\hline \multicolumn{6}{|l|}{ Physician affiliation } \\
\hline PHC & $0.9(0.3)$ & 10.9 & & $2.5(1.5-4.4)$ & 0.001 \\
\hline $\mathrm{OHS}$ & $0.9(0.5)$ & 3.1 & & $2.5(0.9-7.1)$ & \\
\hline Private & $-0.03(0.5)$ & 0.0 & & $1.0(0.4-2.5)$ & \\
\hline Hospital & & & & 1 & \\
\hline
\end{tabular}

$\mathrm{N}=465$ (due to missing information on diagnosis and affiliation). Nagelkerke's $\mathrm{R}^{2}$ was $0.285 ; 77.4 \%$ predicted percentage correct. $P$-values are provided for $\beta$-coefficients.

Abbreviations: SE, standard error; OR, odds ratio; CI, confidence interval; MSD, musculoskeletal diseases; MD, mental disorders; Other, other diagnostic groups; CR, cardiorespiratory diseases; PHC, primary health care; OHS, occupational health service. 